INPLASY

PROTOCOL

To cite: Oliveira et al.

Reference values for external and internal load monitoring in professional male soccer players: A systematic review. Inplasy protocol 202180057. doi:

10.37766/inplasy2021.8.0057

Received: 14 August 2021

Published: 14 August 2021

Corresponding author:

Rafael Oliveira

rafaeloliveira@esdrm.ipsantarem.pt

Author Affiliation:

Sports Science School of Rio Maior-Polytechnic Institute of Santarém

Support: None.

Review Stage at time of this submission: Data extraction.

Conflicts of interest:

None declared.

\section{Reference values for external and internal load monitoring in professional male soccer players: A systematic review}

Oliveira, R1; Martins, $A^{2}$; Moreno-Villanueva, $A^{3}$; Brito, JP4; Nalha, M5; Rico-González, M6; Clemente, FC7.

Review question / Objective: The current systematic review purposed to (1) identify and summarize studies that have examined external and internal training load monitoring and (2) to provide references values for the main measures for professional male soccer players.

Condition being studied: Through this systematic review, external and internal workload measures will be analysed and described for the different days of the week. With such information, coaches, their staff and practioners will be able to collect reference values of the main external and internal measures for professional male soccer players.

Information sources: FECYT (MEDLINE, Scielo, and Web of Science), PubMed, and Scopus.

INPLASY registration number: This protocol was registered with the International Platform of Registered Systematic Review and Meta-Analysis Protocols (INPLASY) on 14 August 2021 and was last updated on 14 August 2021 (registration number INPLASY202180057).

\section{INTRODUCTION}

Review question / Objective: The current systematic review purposed to (1) identify and summarize studies that have examined external and internal training load monitoring and (2) to provide references values for the main measures for professional male soccer players.

Condition being studied: Through this systematic review, external and internal workload measures will be analysed and described for the different days of the week. With such information, coaches, 
their staff and practioners will be able to collect reference values of the main external and internal measures for professional male soccer players.

\section{METHODS}

Participant or population: Healthy professional male soccer players.

Intervention: Exposure to entire training sessions for number of weeks and sessions included (minimum one week).

Comparator: Not required. Eventually, comparisons between playing positions and/or competitive levels within the same age-group and/or age-groups.

Study designs to be included: No restrictions imposed on study design.

Eligibility criteria: Population: Healthy professional male soccer players. Intervention: Exposure to entire training sessions for number of weeks and sessions included (minimum one week). Comparator: Not required. Eventually, comparisons between playing positions and/or competitive levels within the same age-group and/or age-groups. Outcomes: Presents at least of one measure among the included in internal load (e.g., heart rate, rate of perceived exertion) and/or external load (e.g., distances covered at different speed thresholds, accelerationbased measures) in absolute values. Whenever relative values allow to calculate absolute values, the study will be included. Study design: No restrictions imposed on study design. Only original and full-text studies written in English.

Information sources: FECYT (MEDLINE, Scielo, and Web of Science), PubMed, and Scopus.

Main outcome(s): Presents at least of one measure among the included in internal load (e.g., heart rate, rate of perceived exertion) and/or external load (e.g., distances covered at different speed thresholds, acceleration-based measures) in absolute values. Whenever relative values allow to calculate absolute values, the study will be included.

Quality assessment / Risk of bias analysis: The methodological quality was assessed using STROBE statement (Von Elm et al., 2008). This checklist has already been used in previous reviews, with proven precision for evaluation of observational cohort studies, case-control studies, and crosssectional studies (Falck, Davis, \& LiuAmbrose, 2017; A. F. Silva, Conte, \& Clemente, 2020). Studies were classified as high quality when they lacked three criteria from the STROBE checklist, while low quality studies were defined as those in which three or more criteria were missing (A. F. Silva et al., 2020). It included 22 items: title of the article and abstract interlinked (item 1), introduction (items 2 and 3), methods (items 4 to 12), results (items 13 to 17 ), discussion (items 18 to 21), and any other information (item 22). Four items were specific to the study design: participants (item 6), variables (item 12), descriptive data (item 14), and outcome data (item 15). The quality assessment was based on the attribution of one point for each checklist item if the criteria were evaluated as being complete (1 point), or incomplete ( 0 points). The sum of the total points counted was divided by the maximum possible (22 items).

Strategy of data synthesis: The following information was extracted from the included original articles: characteristics of the participants (e.g. age; number; competitive level); study duration; study type; internal measures; external measures. In addition, mean and standard deviation or range values (min-max) for the external and internal measures were extracted by overall team and/or by player positions/status.

Subgroup analysis: None.

Sensitivity analysis: None.

Language: Only original and full-text studies written in English.

Country(ies) involved: Portugal, Spain. 
Keywords: football; load quantification; training load; workload.

Contributions of each author:

Author 1 - Rafael Oliveira - The author lead the project, designing the review, coordinate the project and wrote and revised the original manuscript.

Email: rafaeloliveira@esdrm.ipsantarem.pt Author 2 - Alexandre Martins - The author wrote and revised the original manuscript.

Email: alexandremartins@esdrm.ipsantarem.pt Author 3 - Adrián Moreno-Villanueva - The author run the data search, methodological assssment and revised the original manuscript.

Email: more_adri@hotmail.com

Author 4 - João Brito - The author wrote and revised the original manuscript.

Email: jbrito@esdrm.ipsantarem.pt

Author 5 - Matilde Nalha - The author wrote and revised the original manuscript.

Email: matildenalha@gmail.com

Author 6 - Markel Rico-González - The author run the data search, methodological assssment and revised the original manuscript.

Email: markeluniv@gmail.com

Author 7 - Filipe Clemente - The author wrote and revised the original manuscript.

Email: filipe.clemente5@gmail.com 